\title{
DISPERSION EVALUATION OF CARBON NANOTUBE AND MECHANICAL PROPERTIES FOR CARBON NANOTUBE/POLYAMIDE 6
}

\author{
KAZUTO TANAKA, SATOSHI JOTOKU, HIROKI MUKAOKU \& TSUTAO KATAYAMA \\ Department of Biomedical Engineering, Doshisha University, Japan
}

\begin{abstract}
Carbon nanotube (CNT) has excellent mechanical, electrical, and thermal properties, and is anticipated to be used in various fields such as chemical sensors and nanoelectronic devices and for reinforcing nanofillers. Due to its highly cohesive properties, however, it is difficult to make the most of the characteristics of CNT. Recently, the electrospinning method has attracted attention as a method of dispersing CNT. In this study, CNT/polyamide 6 (PA6) composite nanofibers were fabricated by an electrospinning process, and the dispersion of CNT in nanofibers was evaluated. To disperse CNT in CNT/PA6 nanofibers for the electrospinning process, acid treatment was applied to CNT. CNT/PA6 films were made of CNT/polyamide 6 (PA6) composite nanofibers, and their tensile strength was evaluated. The dispersion of treated CNT in the nanofiber was higher than that of untreated CNT. The tensile strength of treated CNT/PA6 film is higher than that of untreated CNT/PA6, and they are both higher than that of PA6 film.

Keywords: carbon nanotube (CNT), nanofiber, Polyamide 6, electrospinning, dispersion, film, tensile strength.
\end{abstract}

\section{INTRODUCTION}

Carbon nanotube (CNT) has unique properties, such as high aspect ratio, high elastic modulus, high electrical conductivity and high thermal conductivity [1], and is anticipated to be used in various fields such as chemical sensors, nanoelectronic devices and reinforcing nanofillers [2], [3]. There are many reports about composite materials in which CNTs are used for the reinforcement of polymer resin [4]-[6]. However, the mechanical property of the composite material is lower than the theoretical predictions from the rules of mixtures [7]. One of the reasons for this lower mechanical property is considered to come from the poor dispersion of CNTs in matrix resin. To improve the dispersion of CNTs, various types of surfactants [8]-[10] and dispersant [11] were used.

The electrospinning method, in which nanofibers are generated as the electrical jet and continuously stretched due to the potential difference between the polymer solution and target, is commonly used for the fabrication of nanofibers of polymers. Through the combination of dispersing of CNTs in the polymer solution and fabrication of nanofibers by electrospinning methods, dispersed CNTs in polymer nanofiber are expected to be obtained.

In this study, CNT/polyamide 6 (PA6) composite nanofibers were fabricated through the electrospinning process and the dispersion of CNT in nanofibers was evaluated. To disperse CNT in CNT/PA6 nanofibers for the electrospinning process, acid treatment was applied to CNT. CNT/PA6 films were made of CNT/PA6 composite nanofibers and their tensile strength was evaluated. 


\section{MATERIALS AND EXPERIMENTAL METHODS}

\subsection{Materials and electrospinning process}

PA6 (Ube Ind., Japan) was the material of the nanofibers. CNT (Showa Denko K.K. Japan, Diameter: $0.15 \mu \mathrm{m}$, length: $6 \mu \mathrm{m}$ ) was used as the filler. Hexa-Fluoro-Iso-Propanol (HFIP) was used as the dissolution solvent. In this study of dispersion of CNT, surface treatment with sulfuric acid/nitric acid $(3: 1 \mathrm{v} / \mathrm{v})$ was used. This acid treated CNT will be known as "treated CNT", and untreated CNT (as received CNT) will be known as "CNT" throughout the paper.

The solution of PA6 was prepared by dissolving 10\% PA6 in a solvent of HFIP, whilst stirring for 90 minutes in a $30^{\circ} \mathrm{C}$ environment. The solution of CNT/PA6 and treated CNT/PA6 was prepared by adding CNT or treated CNT $(1 \%, 5 \%$, and $10 \%$ mass of CNT:PA6) to HFIP, and it was ultra-sonicated. Then, 10\% PA6 was dissolved in the solution whilst stirring for 90 minutes in a $30^{\circ} \mathrm{C}$ environment.

Fig. 1 shows the schematic drawing of the electrospinning process. PA6, CNT/PA6 and treated CNT/PA6 solutions were spun into nanofibers with an electrospinning unit (NEU, Kato tech). A plastic syringe was filled with the solutions and transferred at a flow rate of $1.0 \mathrm{ml} / \mathrm{h}$. The electro-spun nanofibers were collected on a plate covered in aluminum foil. A voltage of $20 \mathrm{kV}$ with a tip-collector distance of $170 \mathrm{~mm}$ at a feeding rate of $0.83 \times 10^{-5} \mathrm{~mm} / \mathrm{sec}(0.05 \mathrm{~mm} / \mathrm{min})$ was applied to fabricate nanofibers.

\subsection{Evaluation of dispersion}

Fabricated nanofibers were observed using a digital microscope (VHX-5000, Keyence) and a transmission electron microscope (TEM, JEM 2100 F, JEOL).

Fig. 2 shows the dispersion evaluation method of CNT in CNT/PA6 composite nanofibers. The distance between CNTs and the average length of CNTs in the nanofibers were measured with the digital microscope and used as indicators of dispersion.

\subsection{Fabrication method of film and its tensile test}

The electro-spun nanofibers were laminated and molded to a film by hot press at $230^{\circ} \mathrm{C}$ with $250 \mathrm{kPa}$ for $30 \mathrm{~seconds}$, with the thickness of $0.1 \mathrm{~mm}$. The following three types of films were used for tensile test: PA6 film, CNT/PA6 film and treated CNT/PA6 film. For CNT/PA6 films and treated CNT/PA6 films, three levels of CNT addition (1\%, 5\% and 10\%) are used.

Fig. 3 shows the tensile test specimen. The specimens were prepared with a punching tool. Tensile tests were conducted by a universal testing machine (EZ Graph, Shimadzu Co., Japan), fitted with a $100 \mathrm{~N}$ load cell at an extension rate of $8.3 \times 10^{-6} \mathrm{~mm} / \mathrm{sec}(0.50 \mathrm{~mm} / \mathrm{min})$. Fracture surfaces of tensile test specimens were observed by scanning electron microscope (SEM JSM-6390LT, JOEL Ltd., Japan) as shown in Fig. 4.

\section{RESULTS AND DISCUSSIONS}

\subsection{Dispersion of CNT}

Fig. 5 shows images of CNT/PA6 nanofibers observed with the digital microscope and TEM. The black line observed by the digital microscope can be confirmed as a CNT by TEM observation. The measured length of CNT is $5.5 \mu \mathrm{m}$, regardless of observation method. The digital microscope can be used for the length measuring tool of CNTs in nanofibers. 


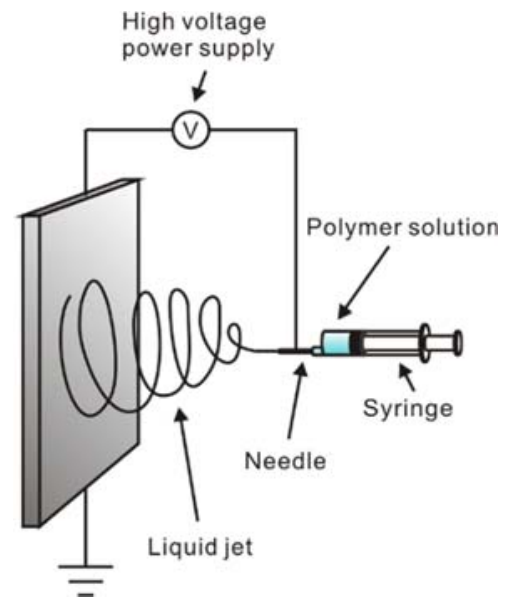

Figure 1: Schematic drawing of the electrospinning process.

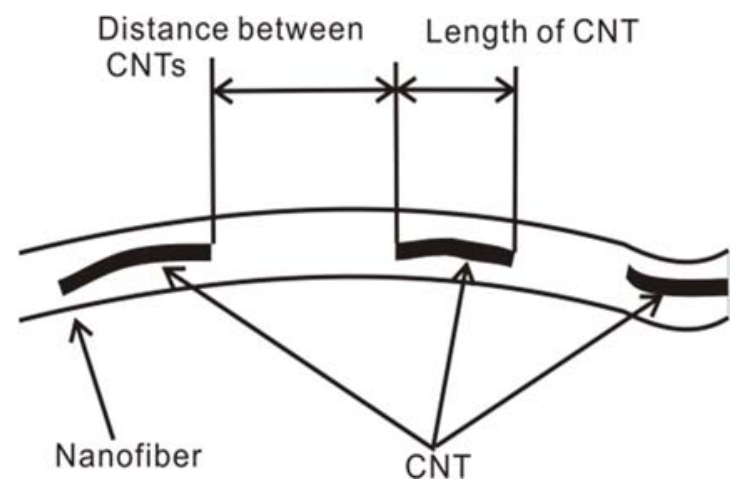

Figure 2: Measured distance between CNTs and CNT length in CNT/PA6 nanofibers.

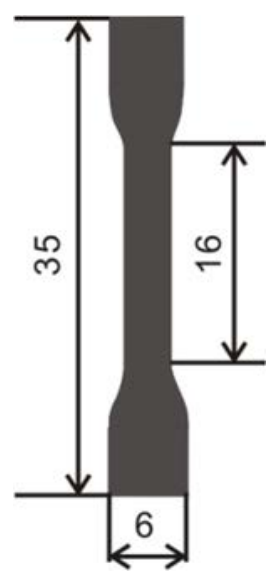

Figure 3: Tensile test specimen. 


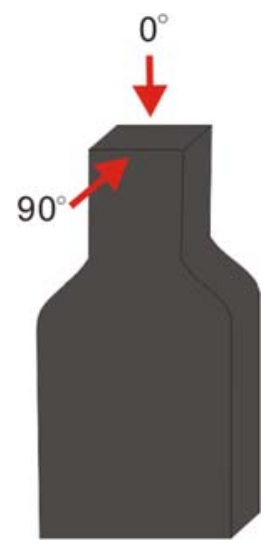

Figure 4: The direction of observation.

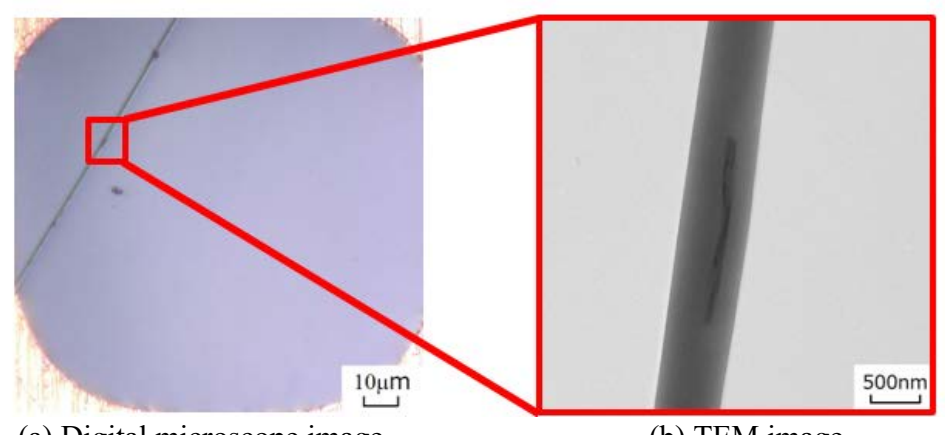

(a) Digital microscope image

(b) TEM image

Figure 5: The images of observing the same position of CNT/PA6 nanofiber using digital microscope and TEM.

Fig. 6 is a histogram of the distance between CNTs measured using the digital microscope. Treated CNT/PA6 nanofiber has a remarkable peak at $10-15 \mu \mathrm{m}$, whereas CNT/PA6 nanofiber has no significant peak. Fig. 7 is a histogram of the average length of CNTs. Treated CNT/PA6 nanofiber has a lower average length of CNT than that of the CNT/PA6 nanofiber. So, the dispersion of treated CNT in the nanofiber is higher than that of CNT. Carboxyl groups are generated on the surface of CNTs through acid treatment, and CNTs are easy to disperse in nanofibers.

\subsection{Tensile test of films}

Fig. 8 shows the stress-strain curves of PA6 films and treated CNT/PA6 films. The strain of treated CNT/PA6 films with high CNT contents is lower than that of PA6. Fig. 9 shows the tensile strengths of PA6, CNT/PA6, and treated CNT/PA6 films. The tensile strength of treated CNT/PA6 films is higher than that of CNT/PA6, and they are both higher than that of PA6 films. Treated CNT/PA6 films with 5\% of CNT produces a $61 \%$ improvement in tensile strength, compared to PA6 film. Ye et al. has reported that the CNT composite fiber had a two-stage reinforcing, bridging and pull-out mechanism [12]. In this study, the dispersion of CNTs in the polymer is considered to contribute to higher tensile strength by increasing the 
crack propagation resistance through the bridging effect, and increasing the pull-outs of CNTs.

Fig. 10 shows the fracture surfaces of CNT/PA6 and treated CNT/PA6 films with 5\% CNT. CNTs at the fracture surface of treated CNT/PA6 films are dispersed, whilst CNTs at the fracture surface of CNT/PA6 films were more condensed. Fig. 11 shows the fracture surfaces of treated CNT/PA6 films. CNTs in films were more condensed as the number of CNTs increased. The condensation of CNTs causes lower tensile strength of treated CNT/PA6 film with $10 \%$ CNT, than that of treated CNT/PA6 film with 5\% CNT.

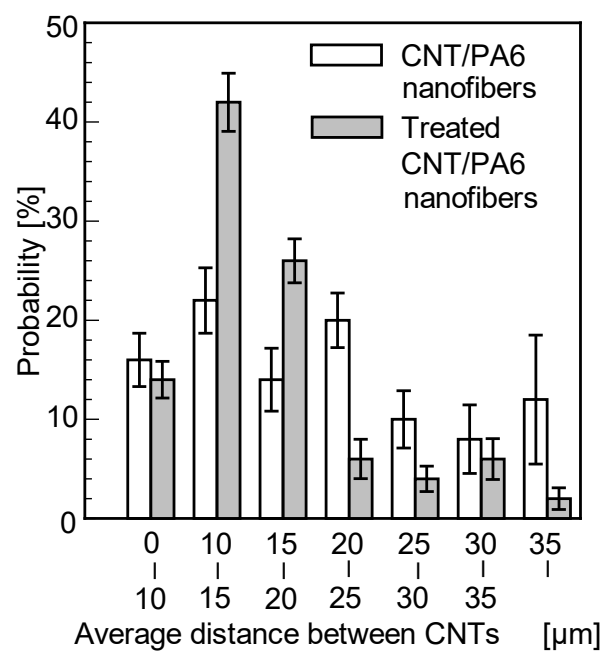

Figure 6: Distribution of average distance between CNTs (CNT 1\%).

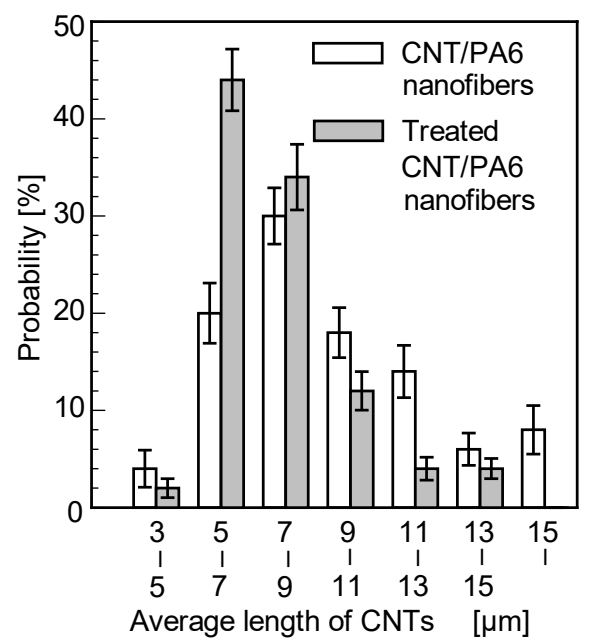

Figure 7: Distribution of average length of CNTs (CNT 1\%). 


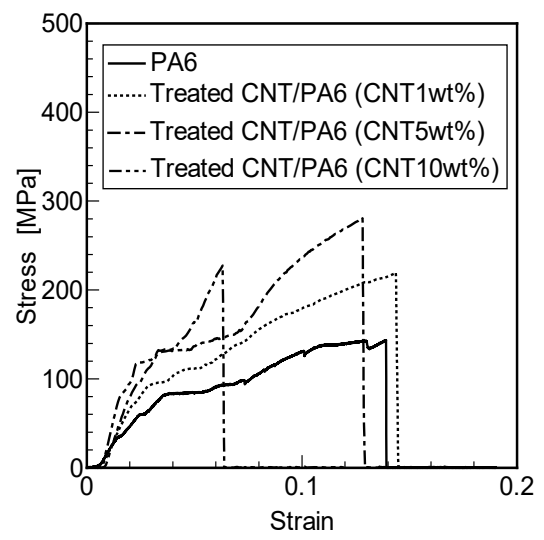

Figure 8: Stress-strain curves.

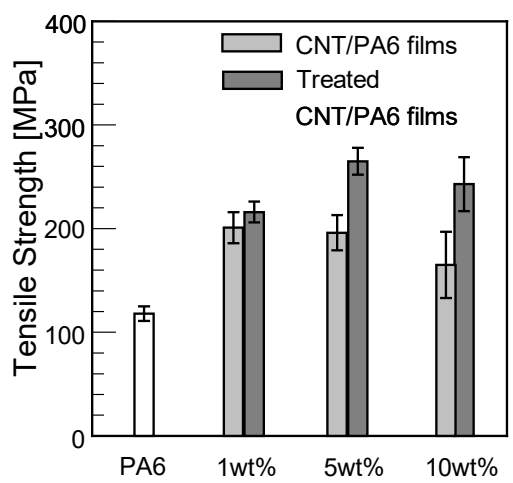

Figure 9: Tensile strengths of PA6, CNT/PA6 and treated CNT/PA6 films.

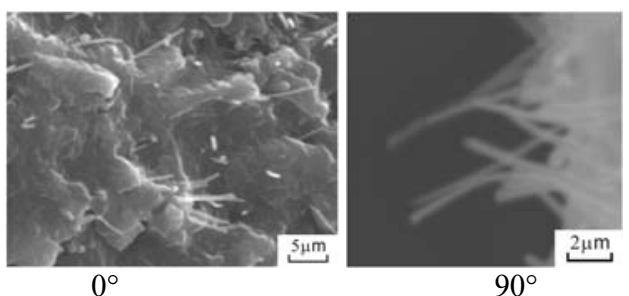

(a) CNT/PA6 films
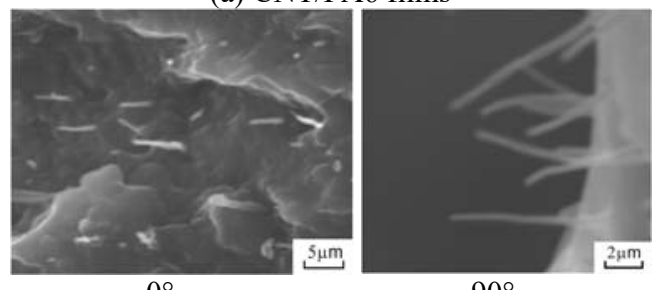

(b) Treated CNT/PA6 films

Figure 10: Observation of cross-section of CNT/PA6 and treated CNT/PA6 films (CNT $5 \%$ ). 

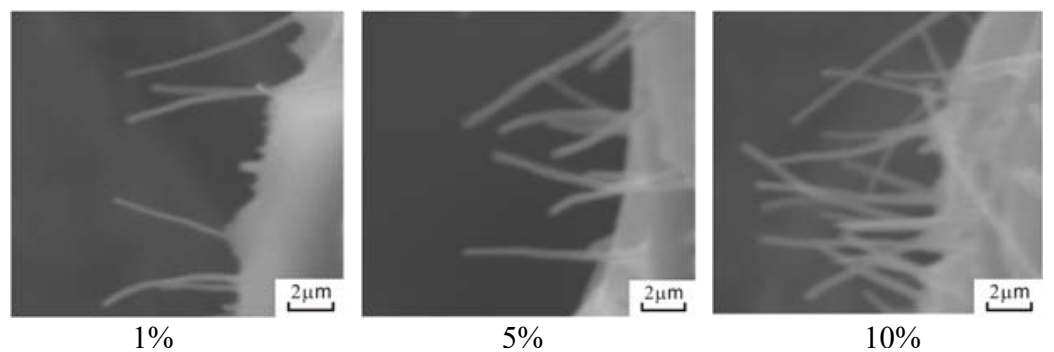

Figure 11: Observation of cross-section of treated CNT/PA6 films.

\section{CONCLUSION}

CNT/polyamide 6 (PA6) composite nanofibers were fabricated by electrospinning process and the dispersion of CNT in nanofibers was evaluated. To disperse CNT in CNT/PA6 nanofibers for the electrospinning process, acid treatment was applied to CNT. CNT/PA6 films were made of CNT/polyamide 6 composite nanofibers, and their tensile strength was evaluated. The investigations yielded the following conclusions:

1. Dispersion of treated CNT in nanofibers was higher than that of CNT. Carboxyl groups were generated on the surface of CNTs by acid treatment, and CNTs became easy to disperse in nanofibers.

2. The tensile strength of treated CNT/PA6 film is higher than that of CNT/PA6 and they are both higher than that of PA6 film.

\section{ACKNOWLEDGEMENTS}

This work was partially supported by a research project on "Research and Development Center for Advanced Composite Materials" of Doshisha University and MEXT (the Ministry of Education, Culture, Sports, Science and Technology, Japan) - Supported Program for the Strategic Research Foundation at Private Universities, 2013-2017: project S1311036.

\section{REFERENCES}

[1] Erik, T., Thostenson, E., Chunyu, L. \& Chou, W.T., Nanocomposites in context. Composites Science and Technology, 65(3-4), pp. 491-516, 2011.

[2] Kumar, B., Castro, M. \& Feller, J.F., Tailoring the chemo-resistive response of selfassembled polysaccharide-CNT sensors by chain conformation at tunnel junctions. Carbon, 50(10), pp. 3627-3634, 2012.

[3] Liu, Y. \& Sujeet, K., Wear performances and wear mechanism study of bulk UHMWPE composites with nacre and CNT fillers and PFPE overcoat. Wear, 300(1-2), pp. 44-54, 2013.

[4] Allaoui, A., Bai, S., Cheng, M.M. \& Bai, B.J., Mechanical and electrical properties of a MWNT/epoxy composite. Composites Science and Technology, 62, pp. 1993-1998, 2002.

[5] Moisala, A., Li, Q., Kinloch, A.I. \& Windle, H.A., Thermal and electrical conductivity of single- and multi-walled carbon nanotube-epoxy composites. Composites Science and Technology and Chemical Physics Letters, 66, pp. 1285-1288, 2006.

[6] Yeha, K., Taib, M. \& Lina, J.Y., Mechanical properties of phenolic-based nanocomposites reinforced by multi-walled carbon nanotubes and carbon fibers. Thin Solid Films, 39(4), pp. 677-684, 2008. 
[7] Deng, F., Ogasawara, T. \& Takeda, N., Tensile properties at different temperature and observation of micro deformation of carbon nanotubes-poly (ether ether ketone) composites. Composites Science and Technology and Chemical Physics Letters, 67, pp. 2959-2964, 2007.

[8] Gojny, H.J., Nastalczyk, J., Roslaniec, Z. \& Schulte, K., Surface modified multiwalled carbon nanotubes in CNT/epoxy-composites. Chemical Physics Letters, 370, pp. 820-824, 2003.

[9] Zhu, J., Kim, J.D., Peng, H., Margrave, L.J., Valery, N. \& Enrique, V., Improving the dispersion and integration of single-walled carbon nanotubes in epoxy composites through functionalization. Nanoletters, 3(8), pp. 1107-1113, 2003.

[10] Zhong, H.W., Li, J. \& Charles, M., Graphitic carbon nanofiber (GCNF)/polymer materials. II. GCNF/epoxy monoliths using reactive oxydianiline linker molecules and the effect of nanofiber reinforcement on curing conditions. Polymer Composites, 26(2), pp. 128-135, 2005.

[11] Gong, X., Liu, J., Baskaran, S., Roger, D. \& Young, S.J., Surfactant-assisted processing of carbon nanotube/polymer composites. Chem. Mater, 12(4), pp 10491052, 2000.

[12] Ye, H., Lam, H., Titchenal, N., Gogotsi, Y. \& Ko, F., Reinforcement and rupture behavior of carbon nanotubes-polymer nanofibers. Applied Physics Letters, 85(10), pp 1775-1777, 2004. 